\title{
An Interference Suppression Method in Wireless Network of C- RAN Architecture
}

\author{
Liu Zhanjun ${ }^{1}$, Li Zhaoyi ${ }^{2}{ }^{2}$, He Hongzhi ${ }^{3}$, Peng Xia ${ }^{4}$ and Zeng Xiaoping ${ }^{5}$ \\ ${ }^{1,2,3,4}$ Chongqing University of Posts and Telecommunications, Chongqing, \\ 400065, China \\ ${ }^{5}$ Chongqing University, Chongqing400044, China \\ liuzj@cqupt.edu.com,Lizhaoyi0608@163.com,408099943@qq.com, \\ 454660432@qq.com
}

\begin{abstract}
The channel sharing in wireless networks leads to user mutual interference, which also exists in the centralized, cooperative, cloud, and clean-radio access network (C-RAN) which is considered as the access architecture of the $5 G$ mobile communication system. In this paper, the problem of uplink interference suppression in the architecture of $C-R A N$ wireless network was investigated. Taking advantage of the centralized processing in the $C-R A N$ architecture, the correlations of the signals from each RRU are analyzed, based on which we proved that under certain condition, interference can be eliminated by utilizing the correlations among signals. According to the theory before, interference matrix between network users was established based on signals from each user to its nearby RRU. Then, the interference cancellation matrix can be gotten. The uplink signals in the network were processed by making use of the interference cancellation matrix, which can turn the interference part caused by the transmit signal of mobile terminal into useful signal.
\end{abstract}

Keywords: C-RAN; centralized processing; interference suppression;

\section{Introduction}

In the wireless communication, the electromagnetic interference among equipment is inevitable because of utilizing the open and shared wireless channels. The interference signals are from the interior of the communication network and are caused by the useful signals from other communication devices, which is different from the noise in the wireless communication. Especially, with the development of the need of wireless communication services, the mobile nodes are more intensive and the node traffic demand is becoming more and more big, which would thus result in the decrease of the cell coverage radius and the distance among mobile terminals. With the reduced distance among the mobile terminals in the adjacent cell, the interference among these terminals is more and more serious. It has significant meaning for increasing the SNR and improving the communication performance to translate the interference energy into useful signal energy.

There are various interference suppression methods in the mobile communication, among which base station pre-coding technique [1-2] and the technique of interference coordination among base stations [3] are the two most typical methods. Base station precoding technique is that user equipment (UE) send uplink sounding pilots to base stations firstly and the base stations estimate the channel based on the accepted uplink sounding pilots [4], then base stations utilize acquired channel-related information to make pre interference suppression code for transmitted signals in order to make the pre-coded signals arrive at user equipment after passing through channels be the same with the 
original signals before pre-coding. This technique requires the coordination of each transmitting terminal and it is relatively easy to implement the techniques like the base station cluster in the downlink communication. However, the pre-coding technique is only applicable to the downlink communication due to the fact that the distributed control of mobile terminals and the difficulty in controlling the transmission waveforms in uplink communication.

The core idea of the inter-cell interference coordination technique is to make certain restriction on the frequency resource of cell edge users to make the frequency of adjacent cell-edge users be orthogonal, and thus achieving the purpose of interference suppression of the cell-edge users. For example, reference [5] proposes an interference coordination method of power adjustment, reference [6] proposes a semi-static interference coordination algorithm based on system loading. These interference suppressions are the methods of preventing interference, instead of eliminating interference among users.

From the above, it can be seen that these two types of interference cancellation mechanisms achieve interference suppression by the idea of avoiding interference. The reason why it is impossible to translate the interference signals of other devices in the network into useful signals is that the receiving devices in the network are managed in distributed mode. However, the C-RAN [6-7] as a wireless access network system has the most obvious characteristic of centralized processing, which is benefit for the interaction between received signals and thus provide a congenital condition for eliminating the interference among mobile users. The network architecture and the system model will be described in detail in section two.

In summary, the existing interference suppression techniques are not applicable to the C-RAN architecture wireless networks. The upstream business will eventually be imported into the baseband resource pool to process, which makes it possible to translate interference signals from different mobile devices in the network into useful signals. Focus on the research of this problem in this paper, a centralized uplink interference suppression technology in C-RAN is proposed.

\section{Introduction of C-RAN}

\subsection{The Wireless Network Architecture of C-RAN}

C-RAN is a radio access network proposed by China Mobile. As shown in Figure 1, CRAN mainly includes three parts:(1)Distributed wireless network comprised of remote radio units(RRU) and antennas; (2) Remote radio units connected by high-bandwidth and low-latency fiber-optical or optical transport network; (3)Centralized baseband resource pool comprised of high-performance general-purpose processor and real-time virtual technology. From this network structure, virtual base station clusters are the controlling center the whole network. Therefore, the virtual base station clusters have all sending signals, receiving signals and channel resource information of the remote radio. Which control all the remote radio frequency in the network. With the centralized scheme, the virtual BS clusters dusters could provide interference cancellation in uplink transmission by inverse matrix reception [9-10] Without information between the RRUs in the Network. 


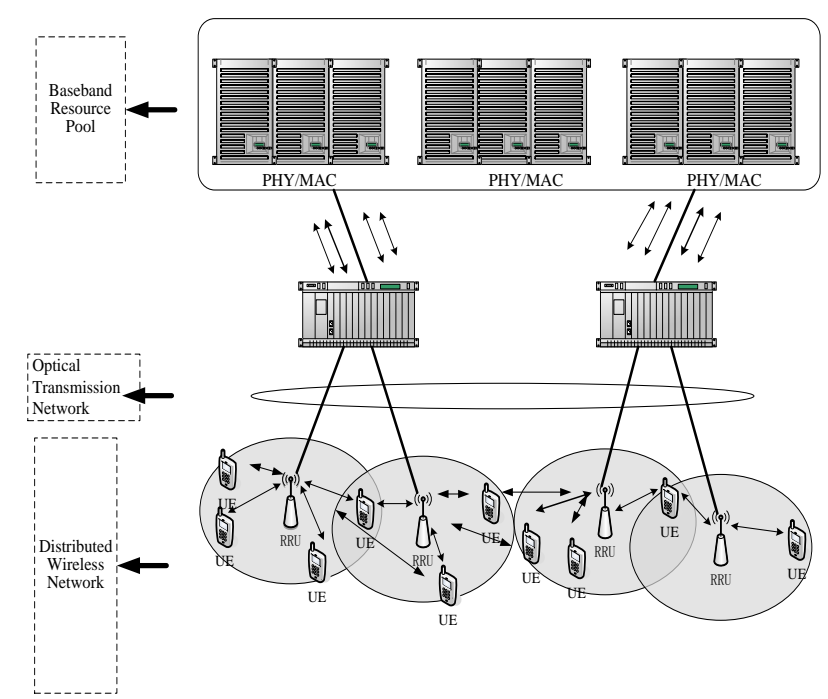

Figure 1. The System of C-RAN Wireless Access Network Architecture

\subsection{Network Model}

This research is mainly based on TDD (time division duplexing) mode. Assuming the system has N RRUs and M UEs, the signal received by each RRU is comprised of signals transmitted by each UE and background noise. The transceiver conditions of the entire system are as follow:

$$
\begin{gathered}
f_{r_{-} s 1}(t)=f_{s 1}(t) * h_{1, s 1_{-} R R U}(t)+f_{s 2}(t) * h_{2, s 1_{-} R R U}(t)+\mathrm{L}+f_{s M}(t) * h_{M, s 1_{-} R R U}(t)+n_{1}(t) \\
\mathrm{M} \\
f_{r_{-} s i}(t)=f_{s 1}(t) * h_{1, s i_{-} R R U}(t)+f_{s 2}(t) * h_{2, s i_{-} R R U}(t)+\mathrm{L}+f_{s M}(t) * h_{M, s i_{-} R R U}(t)+n_{i}(t) \\
\mathrm{M} \\
f_{r_{-} s M}(t)=f_{s 1}(t) * h_{1, s M_{-} R R U}(t)+f_{s 2}(t) * h_{2, s M_{-} R R U}(t)+\mathrm{L}+f_{s M}(t) * h_{M, s M_{-} R R U}(t)+n_{N}(t)
\end{gathered}
$$

Where $f_{s i}(t)$ is the real transmitted signal from $U E_{i}, f_{r_{-} s i}(t)$ is the actual signal received by RRU which communicates with $U E_{i} . h_{i, s j_{-} R R U}(t)$ is channel response from $U E_{i}$ to RRU which communicates with $U E_{j} . n_{1}(t) \ldots n_{i}(t) \ldots n_{N}(t)$ are independent additive white Gaussian noises with zero mean and $N_{0}$ variance. “*” means convolution.

Accordingly, the transceiver conditions of the entire system in frequency domain are as follow:

$$
\mathrm{F}_{\mathrm{r}}(\mathbf{w})=\mathbf{H}(\mathbf{w}) \mathrm{F}_{\mathrm{s}}(\mathbf{w})+\mathrm{N}(\mathbf{w})
$$

Where: 


$$
\begin{aligned}
& \mathbf{F}_{\mathbf{s}}(\mathbf{w})=\left[\begin{array}{c}
f_{s 1}(w) \\
\vdots \\
f_{s i}(w) \\
\vdots \\
f_{s M}(w)
\end{array}\right] \quad \mathbf{F}_{\mathbf{r}}(\mathbf{w})=\left[\begin{array}{c}
f_{r_{-} s 1}(w) \\
\vdots \\
f_{r_{-} s i}(w) \\
\vdots \\
f_{r_{-} s M}(w)
\end{array}\right]
\end{aligned}
$$

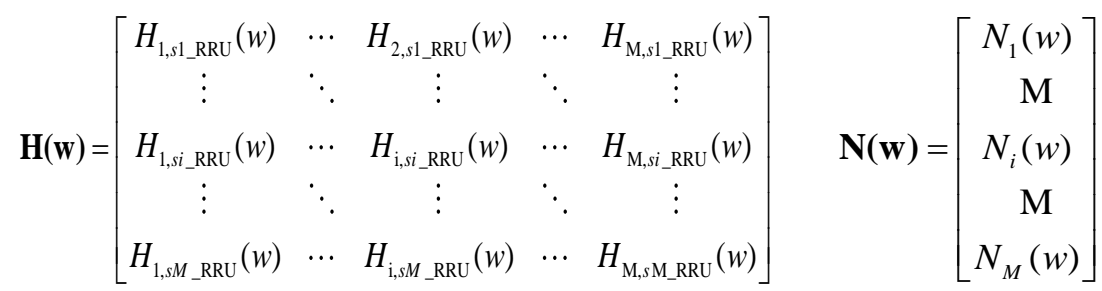

Where $f_{s i}(w), f_{r_{-} s i}(w), H_{i, s_{-} R R U}(w), N_{i}(w)$ are Fourier transformation of $f_{s i}(t)$, $f_{r_{-} s i}(t), h_{i, s j_{-} R R U}(t), n_{i}(t)$ respectively.

\section{Proof of the Existence of Interference Cancellation Matrix (ICM)}

The definition of the interference between transmitted signal and received signal is

$$
\left\|f\left[\mathbf{F}_{\mathbf{r}}(\mathbf{w})\right]-\mathbf{F}_{\mathbf{s}}(\mathbf{w})\right\|
$$

Where $f[$.$] indicates that the received signal is processed by receiver and different$ functions represent different ways to eliminate interference. " $\|$ " represents norm.

If the receive mode is the direct amplification reception (DAR), that is

$$
f\left[\mathbf{F}_{\mathbf{r}}(\mathbf{w})\right]=K \mathbf{F}_{\mathbf{r}}(\mathbf{w})
$$

Where $K$ is an amplification factor.

If there exits a matrix $E$ such that:

$$
\left\|\mathbf{E F}_{\mathbf{r}}(\mathbf{w})-\mathbf{F}_{\mathbf{s}}(\mathbf{w})\right\|<\left\|K \mathbf{F}_{\mathbf{r}}(\mathbf{w})-\mathbf{F}_{\mathbf{s}}(\mathbf{w})\right\|
$$

It indicates that the performance of the received signal after the processing of $\mathrm{E}$ is better than that of the direct amplification reception.

The right side of Equation (5) can be written as:

$$
\left\|K \mathbf{F}_{\mathbf{r}}(\mathbf{w})-\mathbf{F}_{\mathbf{s}}(\mathbf{w})\right\|=\left\|K \mathbf{H}(\mathbf{w}) \mathbf{F}_{\mathbf{s}}(\mathbf{w})+K \mathbf{N}(\mathbf{w})-\mathbf{F}_{\mathbf{s}}(\mathbf{w})\right\|
$$

Merging the right side of Equation (6), we have:

$$
\begin{aligned}
& \left\|K \mathbf{F}_{\mathbf{r}}(\mathbf{w})-\mathbf{F}_{\mathbf{s}}(\mathbf{w})\right\| \\
& =\left\|(K \mathbf{H}(\mathbf{w})-\mathbf{I}) \mathbf{F}_{\mathbf{s}}(\mathbf{w})+K \mathbf{N}(\mathbf{w})\right\|
\end{aligned}
$$

Where $\mathbf{I}$ is a M-order unit matrix. Since the noise signal and the transmission signal are completely independent, that is they are orthogonal. Therefore Equation (7) can be converted to:

$$
\begin{aligned}
& \left\|K \mathbf{F}_{\mathbf{r}}(\mathbf{w})-\mathbf{F}_{\mathbf{s}}(\mathbf{w})\right\| \\
& =\left\|(K \mathbf{H}(\mathbf{w})-\mathbf{I}) \mathbf{F}_{\mathbf{s}}(\mathbf{w})\right\|+K\|\mathbf{N}(\mathbf{w})\|
\end{aligned}
$$

Substituting Equation (2)to the left side of eq.(5), we can get:

$$
\left\|\mathbf{E F}_{\mathbf{r}}(\mathbf{w})-\mathbf{F}_{\mathbf{s}}(\mathbf{w})\right\|=\left\|\mathbf{E}\left[\mathbf{H}(\mathbf{w}) \mathbf{F}_{\mathbf{s}}(\mathbf{w})+\mathbf{N}(\mathbf{w})\right]-\mathbf{F}_{\mathbf{s}}(\mathbf{w})\right\|
$$

The right side of Equation (9) can be simplified to: 


$$
\left\|\mathbf{E F}_{\mathbf{r}}(\mathbf{w})-\mathbf{F}_{\mathbf{s}}(\mathbf{w})\right\|=\left\|(\mathbf{E H}(\mathbf{w})-\mathbf{I}) \mathbf{F}_{\mathbf{s}}(\mathbf{w})+\mathbf{E N}(\mathbf{w})\right\|
$$

Clearly, Equation (10) is equivalent to:

$$
\left\|\mathbf{E F}_{\mathbf{r}}(\mathbf{w})-\mathbf{F}_{\mathbf{s}}(\mathbf{w})\right\|<\left\|(\mathbf{E H}(\mathbf{w})-\mathbf{I}) \mathbf{F}_{\mathbf{s}}(\mathbf{w})\right\|+\|\mathbf{E N}(\mathbf{w})\|
$$

Substituting Equation (8) and Equation (11) to Equation (5), we can obtain:

$$
\begin{aligned}
& \left\|(\mathbf{E H}(\mathbf{w})-\mathbf{I}) \mathbf{F}_{\mathbf{s}}(\mathbf{w})\right\|+\|\mathbf{E N}(\mathbf{w})\| \\
& <\left\|(K \mathbf{H}(\mathbf{w})-\mathbf{I}) \mathbf{F}_{\mathbf{s}}(\mathbf{w})\right\|+K\|\mathbf{N}(\mathbf{w})\|
\end{aligned}
$$

When $\mathbf{E}=\mathbf{H}^{\mathbf{- 1}}$,

$$
\left\|(\mathbf{E H}(\mathbf{w})-\mathbf{I}) \mathbf{F}_{\mathbf{s}}(\mathbf{w})\right\|=0
$$

According to Equation (13), Equation (12) can be written as:

$$
\|\mathbf{E N}(\mathbf{w})\|<\left\|(K \mathbf{H}(\mathbf{w})-\mathbf{I}) \mathbf{F}_{\mathbf{s}}(\mathbf{w})\right\|+K\|\mathbf{N}(\mathbf{w})\|
$$

That is when $\mathbf{E}=\mathbf{H}^{-1}$

$$
\|\mathbf{E N}(\mathbf{w})\|-K\|\mathbf{N}(\mathbf{w})\|<\left\|(K \mathbf{H}(\mathbf{w})-\mathbf{I}) \mathbf{F}_{\mathbf{s}}(\mathbf{w})\right\|
$$

In the above, it can be observed that if $\mathbf{E}=\mathbf{H}^{-1}$ the matrix $E$ can reduce interference when the increase of interference caused by the matrix $E$ are less than the mutual interference among mobile terminals, at this moment, $\mathbf{E}=\mathbf{H}^{-1}$. The matrix $E$ is defined as ICM. Specifically, this criterion is suitable for the scenario with the interference is serious among users. The interference among mobile terminals are increased with the decrease are serious of the size of the mobile communication network..

\section{The Method of Constructing ICM and Complexity Analysis}

Through the proof above, it shows that when the increase of interference caused by the matrix $\mathrm{E}$ are less than the interference among mobile terminals, it is feasible to improve the system performance by ICM. The benefit from ICM is based on the channel estimation value, e.g ID of UE, ID of RRU. The process of constructing the matrix and the flow diagram of utilizing is shown in Figure 2: 


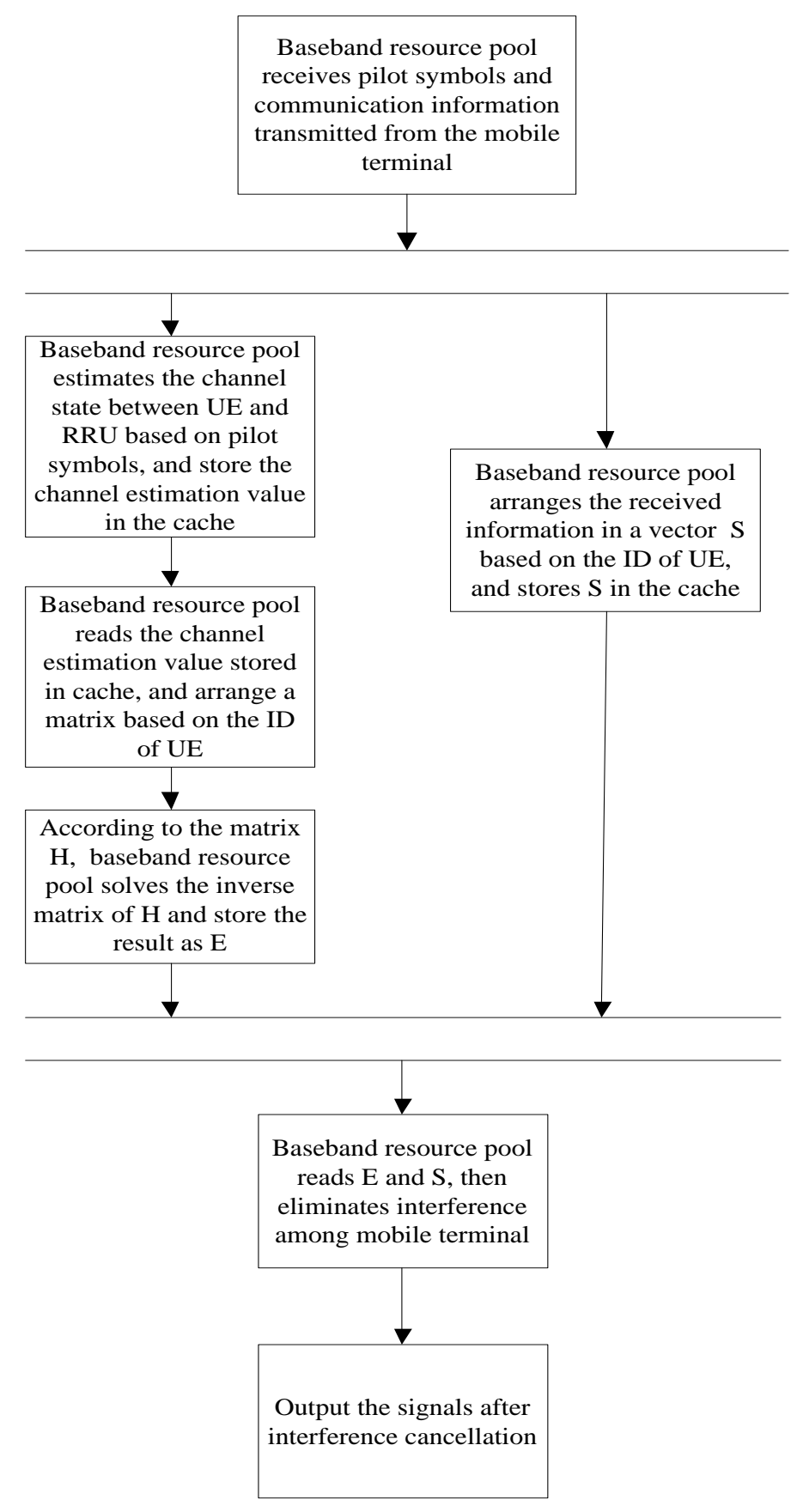

Figure 2. Flowchart of Interference Cancellation

In Figure 2, the baseband resource pool can estimate the channel by adopting the existing channel estimation technique. Then we correspondingly store the ID of UE, the ID of receiving RRU and the channel estimation value into a three-dimensional array. The format of this array is shown in figure 3. Based on the value, the method of constructing matrix is proposed as follows:

\begin{tabular}{|c|c|c|}
\hline The ID of UE & The ID of RRU & $\begin{array}{c}\text { The estimation } \\
\text { from UE to RRU (uplink) }\end{array}$ \\
\hline
\end{tabular}

Figure 3. The Format of Array 
Based on the ID of UE, baseband resource pool reads the vesting information of UE. Based on the vesting information of UE, baseband resource pool reads the ID of RRU that the UE vests in, then baseband resource pool reads all channel estimation value included in the three-dimensional array which include ID of the RRU. And baseband resource pool arranges these channel estimation value to form a row vector in increasing order according to ID of UE.

For those UEs with different IDs, baseband resource pool generates $\mathrm{N}$ row vectors successively in the order of the numbers of those IDs of their corresponding UEs according to the above method. Then baseband resource pool arranges these row vectors successively in a column and forms an $\mathrm{NxN}$ channel state matrix $H$, then stores $H$ in the cache.

Baseband resource pool reads the channel state matrix $H$ stored in the cache, then solves the inverse matrix of $H$, and saves it as $E$. This matrix is namely the interference cancellation matrix.

In Figure2, the method which involved in the usage of interference cancellation matrix is as follows:

Baseband resource pool reads the original signals of UEs from each RRU and arranges these signals in a column vector $m$ in according order of UE ID. Finally, baseband resource pool calculates $X=E \times m$, and outputs the results, which are the transmit signals of UEs with interference cancellation.

When the system has $\mathrm{N}$ users in the uplink communication, the complexity of the traditional direct amplifying receiving algorithm is only depended on the number of the users, which requires $O(N)$ computations. For the proposed algorithm, it needs to construct a $\mathrm{N}$ order interference cancellation matrix to restore the signal vector, causing $\mathrm{N} 2$ times multiplications and $N(N-1)$ addition operations, and thus its complexity is $O\left(N^{2}\right)$. Note that the cloud computing of C-RAN architecture can support massive calculation, thus the increasing complexity of the proposed algorithm due to ICM can be negligible.

\section{Simulation and Analysis}

For the simulation we consider the system with 19 RRUs and 120 UEs and the coverage radius of each RRU is 100 meters. The UEs are distributed randomly within the system, and assigned to each RRU based on the coverage of each RRU. Each UE transmits 4800 binary symbols and each mobile terminal occupies a bandwidth of $180 \mathrm{KHz}$ without consideration of the mobility of UE and channel coding. Assume the 4QAM, 16QAM, 64QAM are used in the baseband modulation respectively. Channel estimation error [10] follows a normal distribution with $N \sim\left(0, \beta^{2}\right)$, where $\beta^{2}$ is the error variance and the channels are independent. According to the literature [11-12], the channel model is set up as follow:

$$
y_{j}=\left(P_{i}\right)^{1 / 2} h_{i j} s_{i}+n_{j}
$$

Where $P_{i}$ is the transmission power of the $U E_{i}$, which was set as $50 \mathrm{~mW}$. $h_{i j}=g_{i j} /\left(d^{3}\right)^{1 / 2}$, where $h_{i j}$ is the channel gain from $U E_{i}$ to $R R U_{j}, d$ is the distance between transmitter and receiver. $g_{i j}=a+j b, a$ and $b$ all obey Gaussian distribution with $N \sim(0,1 / \sqrt{2}) . n_{j}$ is the additive white Gaussian noise with zero mean and $N_{0}$ variance.

Firstly, the network generates the virtual transmitted binary symbols of each UE. With the QAM modulation, then the UE will array the interfering signals from other users and 
Gaussian white noise into column vector. Further-more, based on the interference cancellation matrix the signal column vector, form which the processed signal column vector is obtained. Then adopt QAM demodulation to these signals and get binary symbols of each UE. Finally calculate the BER by comparing these symbols with the original binary symbols transmitted by each UE.

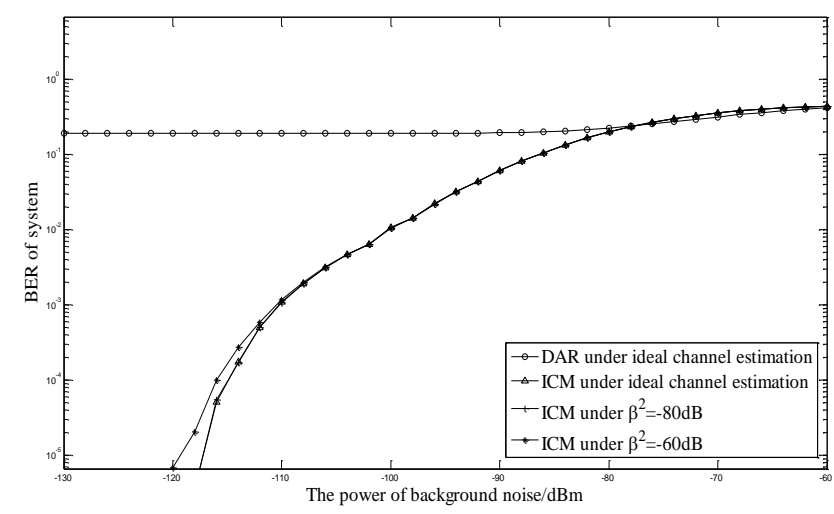

Figure 4. Background Noise and Bit Error Rate under 4QAM Modulation

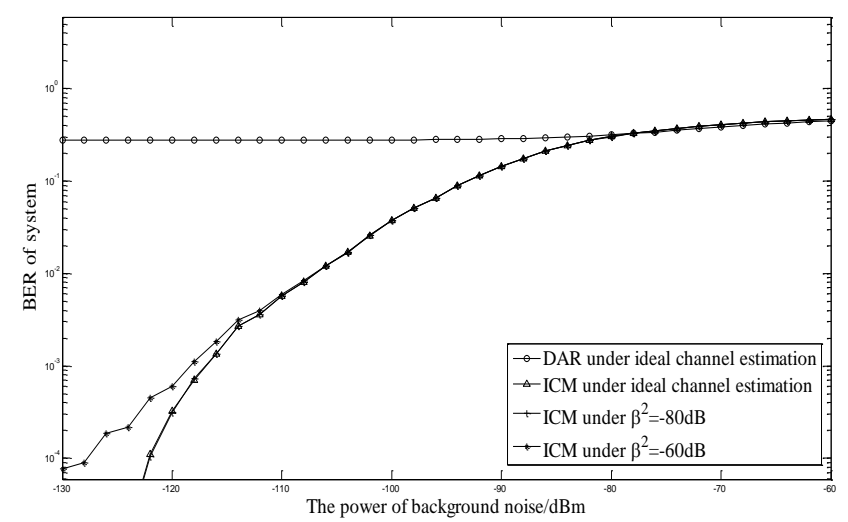

Figure 5. Background Noise and Bit Error Rate under 16QAM Modulation

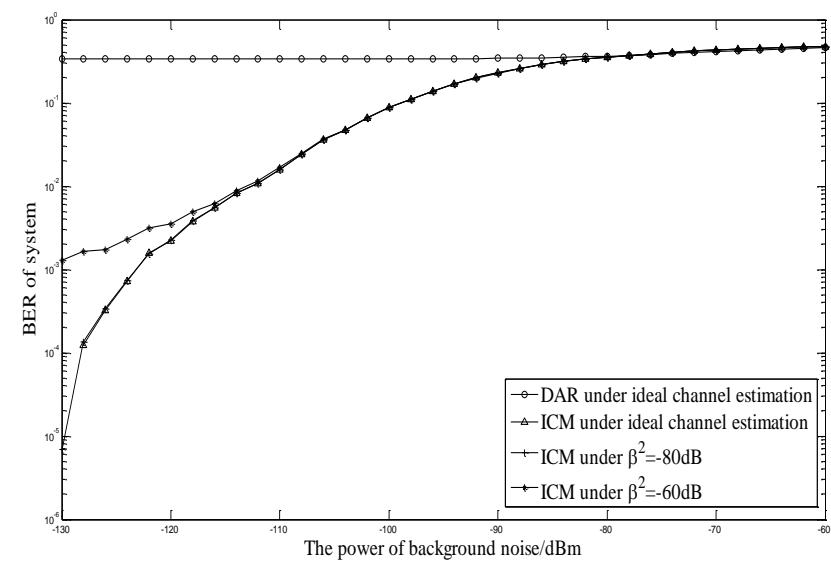

Figure 6. Background Noise and Bit Error Rate under 64QAM Modulation 
Figure 3 -Figure 5 represents the Background Noise\&BER of received signals adopting 4QAM, 16QAM, 64QAM respectively. It can be seen from the figure3, figure4, figure5 that the ICM has considerable performance improvement compared to the DAR (direct amplification reception). Specifically, we can obtain the best with ICM under ideal channel estimation. When the channel estimation is ideal, the performance of ICM reaches the peak. When the variance of channel estimation error increases, the performance of ICM has declined, but still obviously surpasses that of the direct amplification reception under ideal channel estimation. In addition, with the decreasing of the background noise, the system BERs of the two receiving modes are reducing. The system BER of the ICM decreases more quickly, whereas the system BER of the direct amplification reception tends to converge quickly, which due to the influence to BER from uncertain of the interference among mobile terminals.

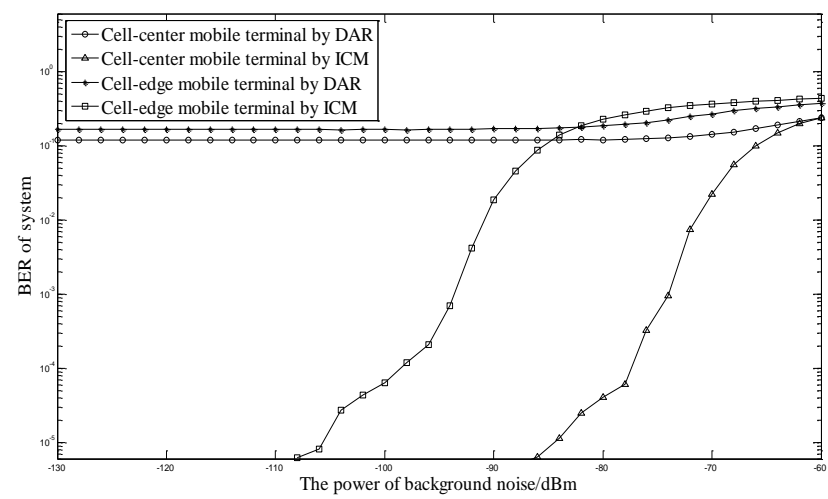

\section{Figure 7. Background Noise-BER of cell-center UE And cell-edge UE in Cell One Under 64QAM}

In the system, because the location of the user in cell is different and path loss is not the same, these factors lead to the difference of users call quality. We can see from Figure 6 , as the decrease of background noise, compared to the direct amplification reception, the interference cancellation matrix can not only improve the performance of users which in central cell, but also can improve the ones at the border of cell. Therefore, the interference cancellation matrix can improve the QoS of all users in the net.

\section{Conclusion}

Focus on the research of the uplink channel interference in the C-RAN architecture wireless network, the characteristics of centralized processing of the C-RAN architecture wireless network makes it is possible to achieve the centralized interference cancellation of uplink. The interference between uplink signals has a correlation to each other (there is a correlation between the interference of uplink signals) under C-RAN network architecture by theoretical analysis. And it is proved that when the background noise is small, there would be a matrix which make operation on the uplink signal, namely to utilize the correlation between signals to convert partial interference signal to useful signal, which provides a theoretical basis for the proposing of the interference cancellation matrix. However, by this way the background noise would be amplified too.

Simulation analysis is carried out according to the method. The modulations of 4QAM, 16QAM, 64QAM were used in the simulation. The performance such as bit error rate of received signal under different noise background is analyzed at the receiver, the simulation results show that, when the background noise is small, the bit error rate of proposed method is obviously less than that of traditional reception way, which infers/proves that the proposed method can improve 
the interference cancellation performance, to some extent. But with the increase of background noise, the received bit error rate of the proposed method increased gradually.

\section{Acknowledgement}

This work was supported by National Natural Science Foundation of China (NSFC) (61401053) and Program for Changjiang Scholars and Innovative Research Team in University (IRT1299).

\section{References}

[1] W. Guoxing, "Reserch of Interference Suppression Technologies in the Next Generation Wireless Communication System", (2010).

[2] J. Han and K. Zhou, "Interference research and analysis of LTE-R, IEEE 5th International Symposium on MAPE", (2013).

[3] P. Liu and Z. Xu, "Blind MMSE-Constrained Multiuser Detection", IEEE Transactions on Vehicular Technology, (2008).

[4] M. Kountouris and R. W. Heath, C. Chan-Byoung and T. Salzer, "Shifting the MIMO Paradigm", IEEE Signal Processing Magazine, vol. 24, no. 3, (2007), pp. 36-46.

[5] M. Matthaiou, P. de Kerret, G. K. Karagiannidis and J. A. Nossek, "Mutual Information Statistics and Beamforming Performance Analysis of Optimized LoS MIMO Systems", IEEE Transactions on communications, vol. 58, no. 11, (2010), pp. 3316-3329.

[6] X. Xianzhong, Z. Pinlian and Q. Gang, "Delay-Tolerance SLNR Precoding to Mitigate Inter-Cell Asynchronous Interference", Journal of Jilin University (Information Science Edition), vol. 28, no. 1, (2010), pp. 1-7.

[7] C. Huppert and J. G. Klotz, "Required Transmit Power Applying Tomlinson-Harashima-Precoding in Scalar and MIMO Broadcast Systems", IEEE Transactions on communications, vol. 58, no. 10, (2010), pp. 3011-3022.

[8] A. Razi, D. J. Ryan, I. B. Collings and Jinhong Yuan, "Sum Rates, Rate Allocation and User Scheduling for Multi-User MIMO Vector Perturbation Precoding", IEEE Transactions on Wireless Communications, vol. 9, no. 1, (2010), pp. 356-367.

[9] M. S. Kang and B. C. Jung, "Decentralized Inter-cell Interference Coordination in Uplink Cellular Networks using Adaptive Sub-band Exclusion", IEEE Wireless Communications and Networking Conference, (2009).

[10] 3GPP, TS 36.423 V8.6.0, Requirements for Evolved UTRAN (E-UTRAN) and X2 application protocol (X2AP), (2009).

[11] G. Zhang and C. Zhang, "A Novel Uplink Interference Coordination Scheme Using High Interference Indicator", IEEE Vehicular Technology Conference (VTC) fall, (2010), Ottawa, Canada.

[12] C. U. Castellanos, F. D. Calabrese, K. I. Pedersen and C. Rosa, "Uplink Interference Control in UTRAN LTE Based on the Overload Indicator", IEEE Vehicular Technology Conference (VTC)fall, (2008).

\section{Authors}

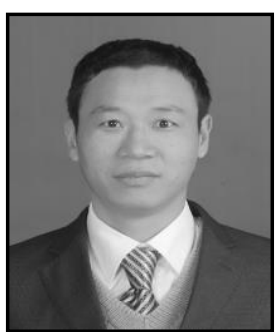

Liu Zhanjun, he was born in Hebei China in 1975, received his Bachelor degree from Hebei Normal University in 2001, received his Master degree form Chongqing University of Posts and Telecommunications in 2004, and now associate professor of Chongqing University of Posts and Telecommunications. His interesting study field is wireless communication networks, mobile telecommunication.

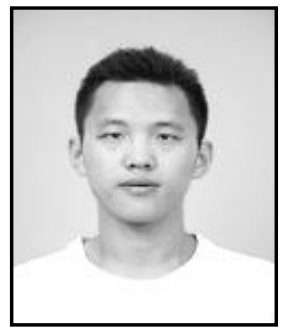

Li Zhaoyi, he was born in Hebei in June 1993, received his Bachelor degree from Industrial and Commercial college, Hebei university in 2015, and now is a master student in Chongqing University of Posts and Telecommunications. His interesting study field is wireless communication networks. 

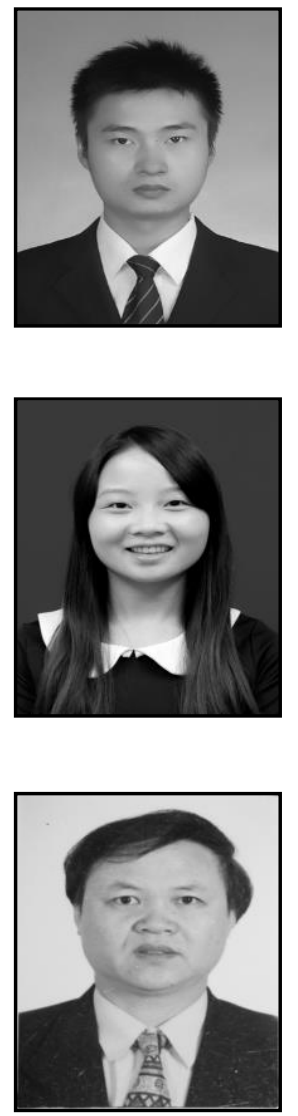

He Hongzhi, he was born in Chongqing in October 1989, received his Bachelor degree from Chongqing Three Georges University in 2012, and now is a master student in Chongqing University of Posts and Telecommunications. His interesting study field is wireless communication networks.

Peng Xia, she was born in Ba'nan Chongqing in April 1991, received her Bachelor degree from Chongqing Three Georges University in 2013, and now is a master student in Chongqing University of Posts and Telecommunications. Her interesting study field is wireless communication networks.

Zeng Xiaoping, he was born in Sichuan in 1956, received his bachelor degree, Master degree and Doctor degree from Chongqing University in 1982, 1987 and 1996, now professor of Chongqing University, His interesting research fields including wireless communication, mobile communication, and signal processing. 
International Journal of Future Generation Communication and Networking Vol. 9, No. 12 (2016) 